\title{
Time for a change: What dominance durations reveal about adaptation effects in the perception of a bi-stable reversible figure
}

\author{
Thomas C. Toppino • Gerald M. Long
}

Published online: 19 December 2014

(C) The Psychonomic Society, Inc. 2014

\begin{abstract}
The effect of adaptation on the perception of a reversible figure was examined in the context of the socalled "reverse-bias effect" in which prolonged exposure to an unambiguous version of a bi-stable ambiguous stimulus serves to bias an observer to report the alternative version of the subsequently viewed ambiguous stimulus. Exposure to the unambiguous stimulus presumably selectively adapts and weakens the neural structures underlying that particular interpretation of the ambiguous figure. We extended previous research by examining the dominance durations for the two alternatives of the reversible figure (i.e., how long each alternative was perceived when it was dominant) in addition to the measures of response rate and choice preference used by other researchers. We replicated earlier findings with the previously used measures. Interestingly, adaptation with an unambiguous version of the ambiguous stimulus produced an asymmetrical effect on the dominance durations of the subsequently presented ambiguous stimulus, relative to a no-adaptation control. The dominance durations were lengthened for the perceptual organization that was the opposite of the adaptation stimulus while they were relatively unaffected for the perceptual organization that was the same as the adaptation stimulus. Our findings are consistent with the argument that adaptation effects play an important role in perceptual bistability. The asymmetrical dominance-duration findings further suggest that adaptation operates in a perceptual system in which the alternative perceptual representations of an ambiguous figure reciprocally inhibit one another via cross-inhibitory processes, consistent with views developed in other forms of bistable perception (e.g., binocular rivalry).
\end{abstract}

\footnotetext{
T. C. Toppino $(\bowtie) \cdot$ G. M. Long

Department of Psychology, Villanova University, 800 Lancaster

Avenue, Villanova, PA 19085, USA

e-mail: thomas.toppino@villanova.edu
}

Keywords Adaptation and Aftereffects · Binocular vision: Rivalry/Bistable Perception

\section{Introduction}

Reversible or multi-stable figures are ambiguous visual patterns with the striking characteristic that, during a period of extended viewing, observers' phenomenal experience shifts repeatedly among two or more distinct perceptual interpretations, so that first one and then another perceptual alternative dominates. This class of stimuli has been known since antiquity and has been the object of scientific curiosity and research for the better part of two centuries, since L. A. Necker (1832) introduced the now-famous "Necker cube" (see Fig. 1). Interest in reversible figures has endured because the marked changes in perception that people experience when viewing this type of stimulus occur in the absence of changes in the stimulus itself. This fact suggests to many researchers that reversible figures may be especially useful in elucidating the visual and cognitive processes that are involved in interpreting the often impoverished, incomplete, and ambiguous information in the retinal image and ultimately in revealing the neural basis of conscious visual awareness (Kornmeier \& Bach, 2004; Leopold \& Logothetis, 1999; Toppino \& Long, 2005).

Historically, scientific research with reversible figures has focused on bistable perception, studying figures with two alternative structures; and theoretical accounts of figural reversal have emphasized either the top-down influence of relatively global, high-level, cognitive processes or the bottom-up influence of relatively passive, localized, sensory processes. Top-down processes are implicated by findings that the perception of reversible figures is influenced by expectation (e.g., Bugelski \& Alampay, 1961; Fisher, 1967; Leeper, 1935), knowledge of reversibility (e.g., Girgus, Rock, \& 


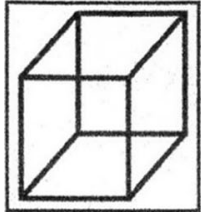

(a)
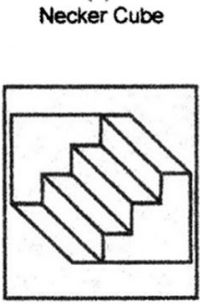

(f)

Schroeder Staircase

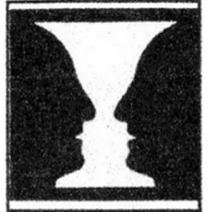

(b)
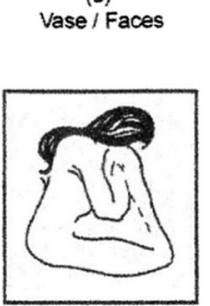

(g) Man / Girl

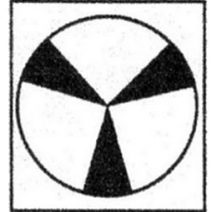

(c)
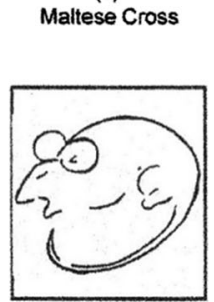
(h)
Rat / Man

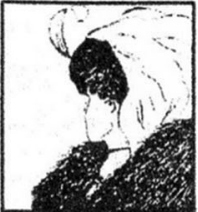

(d)
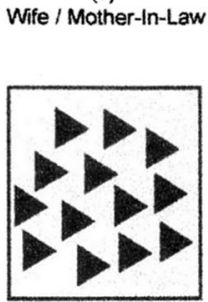

(i)

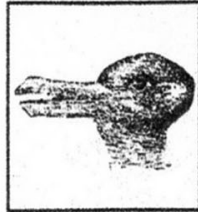

(e)

Duck / Rabbit

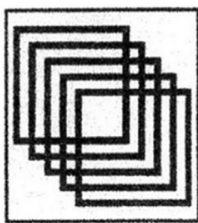

Fig. 1 a-j Examples of well-known reversible figures. a The Necker cube, b Rubin's face/vases figure, c the Maltese cross, d Boring's wife/mother-in-law figure, e the duck/rabbit figure, f Schroeder's

staircase, $\mathbf{g}$ Fisher's man/girl figure, $\mathbf{h}$ the rat/man figure, i Attneave's ambiguous triangles, $\mathbf{j}$ overlapping squares figure. (From Long \& Toppino, 2004)

Egatz, 1977; Rock \& Mitchener, 1992), experience with the particular reversible stimulus (van Ee, van Dam, \& Brouwer, 2005; Long, Toppino, \& Kostenbauder, 1983), and volition (Hochberg \& Peterson, 1987; Struber \& Stadler, 1999; Toppino, 2003; van Ee et al., 2005). Bottom-up processes also are implicated by several lines of evidence. For example, the frequency of reversals is sensitive to low-level stimulus variables (e.g., Babich \& Standing, 1981; Lynn, 1961); and multiple, simultaneously presented versions of the same ambiguous figure can be seen to reverse independently (e.g., Flugel, 1913; Long \& Toppino, 1981; Toppino \& Long, 1987). Perhaps most dramatic is the finding that reversals are dependent on continuous stimulation of the same retinal location, such that moving the ambiguous stimulus to a new retinal region returns the observer's performance back to baseline (e.g., Blake, Sobel, \& Gilroy, 2003; Kohler, 1940; Toppino \& Long, 1987; von Grunau, Wiggin, \& Reed, 1984).

Given the clear evidence for top-down and bottom-up processes, many contemporary researchers have adopted a hybrid approach that recognizes a role for both (e.g., Hochberg \& Peterson, 1987; Kornmeier \& Bach, 2012; Long \& Toppino, 2004; Toppino \& Long, 2005). The ultimate challenge is to understand how lower-order and higher-order processes interact and are integrated in determining perceptual awareness (e.g., Klink et al., 2008a, b; Kornmeier, Pfaffle, \& Bach, 2011; Toppino, 2003). It also, of course, remains critical to identify and characterize specific mechanisms that contribute to figural reversals, regardless of the processing level. In this paper, we investigate the effect of adaptation and related inhibitory processes on the perception of a stationary, twodimensional, reversible figure.

Explanations invoking adaptation are among the most frequently proposed accounts of perceptual multi-stability. Early versions of this hypothesis related perceptual reversals to

neural fatigue or "satiation", and were formulated in the context of now-discredited Gestalt assumptions about the underlying physiology (Kohler \& Wallach, 1944). Subsequent versions of the hypothesis have proposed that exposure to a reversible figure activates two or more mutually exclusive sets of neural structures that compete for dominance (e.g., Blake, Sobel, \& Gilroy, 2003; Nawrot \& Blake, 1989; Toppino \& Long, 1987; Wallis \& Ringelhan, 2013). Some aspect of the neural structures supporting the currently dominant percept is thought to adapt or weaken over time, until the structures underlying an alternate interpretation of the reversible stimulus gain ascendancy, producing a phenomenal reversal. A cyclic process is then thought to ensue in which the structures underlying the currently dominant percept adapt, while those underlying the non-dominant percept recover until the observer experiences another phenomenal reversal (e.g., Dornic, 1967).

A phenomenon that Long and Toppino (2004) termed the "reverse-bias effect" provides particularly strong evidence for the involvement of adaptation processes. In contrast to the well-known finding that observers can be primed to report a particular interpretation of an ambiguous figure by first briefly presenting that same unambiguous version of the figure, prolonged presentation of the same unambiguous stimulus produces the opposite effect. This reverse bias has been studied extensively in the context of bi-stable reversible figures. When observers view an unambiguous version of a reversible figure for an extended period of time and then are shifted to the ambiguous form of the figure, the observers' perceptual interpretation of the bistable figure typically is the opposite of the previously viewed unambiguous stimulus (i.e., a reverse bias is obtained). Exposure to the unambiguous figure presumably adapts the neural structures supporting the corresponding percept so that, when the ambiguous figure is 
presented, these structures cannot compete effectively with the opposing neural structures, and the opposite interpretation of the stimulus is perceived.

The reverse-bias effect has been observed with a variety of reversible figures, including those involving figure-ground ambiguities such as the Maltese cross (Hochberg, 1950), semantic ambiguities such as the rat/man and the young/old woman figures (Long \& Olszweski, 1999), and perspective ambiguities such as the overlapping-squares figure (Long \& Olszweski, 1999) and the Schroder staircase (Virsu, 1975). The phenomenon has been demonstrated in several ways. First, exposure to an unambiguous version of a reversible figure during the adaptation period affects the proportion of time that each alternative is perceived when the ambiguous stimulus subsequently is presented. The interpretation opposite from that of the adaptation stimulus is seen for a greater proportion of the viewing period than is the interpretation that is the same as the adaptation stimulus (Carlson, 1953; Hochberg, 1950; Nawrot \& Blake, 1989; von Grunau, Wiggin, \& Reed, 1984). Second, observers' initial interpretation of the reversible figure tends to be the opposite of the unambiguous adaptation stimulus (Carlson, 1953; Emerson, 1979; Harris, 1980; Long \& Moran, 2007; Long \& Olszweski, 1999; Long, Stewart, \& Glancey, 2002; Long et al., 1992; Petersik, Shepard, \& Malsch, 1984; Virsu, 1975). And, finally, the number of perceived reversals of an ambiguous stimulus is reduced following extended exposure to an unambiguous version of the stimulus (Long \& Moran, 2007; Long \& Olszweski, 1999; Long et al., 1992).

As noted earlier, the adaptation account of bi-stability is based on the assumptions that the neural structures underlying a particular interpretation of a reversible figure adapt or weaken as a function of the time during which that alternative is perceived (or is dominant) and recover as a function of the time during which it is not perceived (or is suppressed). Research on the reverse-bias effect has provided support for these assumptions. Long, Toppino, and Mondin (1992) varied the duration of the unambiguous adaptation stimulus prior to the presentation of a corresponding ambiguous stimulus (either a rotating Necker cube or a stationary overlappingsquares figure). Consistent with the hypothesis that adaptation progresses as a function of time, they found that increasing the adaptation duration produced a decline in the proportion of trials on which the initial perception of the reversible figure was the same as the adaptation stimulus and reduced the frequency with which phenomenal reversals of the ambiguous figure were perceived. ${ }^{1}$ Long and Moran (2007)

\footnotetext{
${ }^{1}$ It is important to note that Long et al. (1992) demonstrated that observers must view the unambiguous stimulus for a relatively long period of time (e.g., $2 \mathrm{~min}$ ) to ensure the level of adaptation necessary to produce the reverse-bias effect. Brief pre-exposure to the unambiguous stimulus may prime its interpretation, producing a same-percept advantage when the reversible figure is viewed subsequently.
}

found that inserting a delay between the presentation of an unambiguous rotating cube and the presentation of an ambiguous rotating Necker cube reduced or eliminated the bias to perceive the opposite interpretation of the ambiguous test figure as well as the tendency for the number of reversals to be suppressed. The delay presumably allowed the adapted structures to recover sufficiently to compete for dominance.

The claimed role for adaptation in figural reversals has not gone unchallenged. Critics of the hypothesis have argued that the process underlying reversals reflects stochastic processes and not neural adaptation. Leopold and Logothetis (1999) reasoned that, according to the adaptation hypothesis, there should be a dependency between successive periods of dominance and suppression of the competing perceptual alternatives. "The longer neurons involved in the competition remain inactive, the stronger they should compete in the next cycle..." (pp. 258). However, there is little empirical support for this relationship. Employing several reversible figures, including the well-known Necker cube and the Schröder staircase, Borsellino, DeMarco, Allazetta, Rinesii, and Bartolini (1972) failed to obtain a significant correlation between the duration of successive percepts. Their data seemed to suggest a random process, with the distribution of percept durations skewed to the right and well-described by a gamma distribution. Other researchers found similar results with reversible figures, although the exact mathematical form of the right-skewed distribution has been questioned (e.g., Taylor \& Aldridge, 1974; Brascamp, van Ee, Pestman, \& van den Berg, 2005). Still others have found similar results using the binocular-rivalry paradigm in which bistable perception occurs as a consequence of different stimuli being projected simultaneously and dichoptically to each eye (e.g., Fox \& Herrmann, 1967; Walker, 1975). In the latter paradigm, observers typically perceive the input to one eye at a time in an alternating pattern that bears many similarities to the experience that observers report when viewing bistable ambiguous stimuli.

The research reviewed above examining the duration of successive percepts strongly suggests that a stochastic process contributes to perceptual reversals. Perhaps this should not be surprising given the ubiquity of noise in the neural system. However, the presence of noise does not necessarily rule out an adaptation process. In a recent study of sequential dependencies between perceptual states, Pastukhov and Braun (2011) obtained clear evidence of adaptation when they used a more sensitive measure than had been used in previous research. Also, a formal demonstration of the compatibility of adaptation and stochastic influences was provided by Lehky (1988) who modified an earlier adaptation-based model of bistable perception proposed by Attneave (1971) by combining the deterministic adaptation component with a random component that added noise to the system. The model yielded simulation results that captured the essential stochastic 
features of bistable perception in binocular rivalry (and ambiguous figures) while retaining a causal role for adaptation. As Lehky noted, his model serves as a counterexample against the contention that the stochastic character of bistable perception is inconsistent with an adaption account. The theoretical compatibility of adaptation and noise (stochastic modulation) has been corroborated subsequently by other formal models of binocular rivalry that have combined both processes (e.g., Laing \& Chow, 2002; Stollenwerk \& Bode, 2003; Wilson, 2003).

Empirical support for the effects of both adaptation and noise in binocular rivalry was provided by Kang and Blake (2010). Consistent with the effect of adaptation, they showed that longer periods of adaptation of the dominant percept reduced the dominance of that percept in the post-adaptation period. However, an analysis of the coefficient of variation (the ratio of the standard deviation and the mean of dominance durations) as a function of adaptation duration suggested that the contribution of noise is greater to the extent that a perceptual alternative has been weakened through adaptation. Hence, they propose an "adaptation-plus-noise account" of the temporal dynamics associated with bi-stable perception.

Although the adaptation and recovery of neural structures underlying the alternative representations of an ambiguous stimulus display seem to be crucial to explaining perceptual bistability, these processes alone do not account for the fact that the competing representations reciprocally inhibit one another. As numerous investigators have argued, the simple fact that only one alternative of an ambiguous stimulus can be seen at a time would appear to demand an underlying system of competing neural structures in which the dominance of one set of structures suppresses the activity of the other (see Long $\&$ Toppino, 2004). An explanation for such reciprocal inhibition seems to reside in the architecture of the perceptual system that governs how the perceptual alternatives associated with ambiguous input are organized and how they interact. This issue has received scant attention in the reversible-figure literature but has been studied extensively in the context of binocular rivalry (e.g., Levelt, 1965). Although a number of different architectures can be conceptualized (e.g., Fox \& Rasche, 1969), the majority of researchers and theorists working with binocular rivalry have concluded that a cross-inhibitory architecture is most appropriate (e.g., Laing \& Chow, 2002; Lehky, 1988; Moreno-Bote, Rinzel, \& Rubin, 2007; Stollenwerk \& Bode, 2003; Wilson, 2003). In this kind of architecture, the competing perceptual alternatives inhibit one another through separate inhibitory pathways that are at least partially independent. Thus, if we assume that P1 and P2 represent the perceptual alternatives associated with input to Eye 1 and to Eye 2, respectively, P1 inhibits P2 through one set of inhibitory pathways, whereas P2 inhibits P1 via another set of inhibitory links.
A crucial finding consistent with a cross-inhibitory architecture was originally summarized in Levelt's (1965) "Proposition II." Levelt and others (e.g., Fox \& Rasche, 1969) manipulated the strength of the input to one eye via changes in contrast or luminance while holding constant the strength of the input to the other eye. They found that the dominance duration (the duration that a percept is dominant or perceived) of the constant eye was inversely related to the strength of the input to the varied eye, whereas the dominance duration of the varied eye was unaffected. The fact that varying input strength to one eye exerted its effect on the other eye suggests that the perceptual alternatives influence one another through at least partially independent pathways, consistent with the expectations of a cross-inhibitory architecture.

Subsequent research found that varying the strength of the input to one eye did have a small effect on the varied eye, although it was confirmed that its primary effect was on the dominance duration of the constant eye (e.g., Bossink, Stalmeier, \& De Weert, 1993; Mueller \& Blake, 1989). More recently, Brascamp, van Ee, Noest, Jacobs, and van den Berg (2006) noted that earlier research had held strength of input to the constant eye at a high level and reduced the strength of the input to the varied eye. Under the same conditions, Brascamp et al. (2006) replicated the classic finding, but they found the reverse when they held input strength constant at a low level and increased the strength of the input to the varied eye. This suggests a revision of Levelt's Proposition II: Varying the strength of the input to one eye primarily affects the dominance durations of the eye with the stronger input. Regardless, however, the asymmetry of the effect is consistent with the hypothesis that a perceptual architecture involving cross-inhibitory processes underlies bistable perception in binocular rivalry.

The alternating perceptions that characterize reversible ambiguous figures also may reflect a cross-inhibitory architecture within the perceptual system. Numerous parallel findings in research involving binocular rivalry and ambiguous stimuli suggest that the two forms of bi-stable perception share important underlying processes (e.g., Leopold \& Logothetis, 1999). However, considerable caution should be exercised in generalizing the results and conclusions from one empirical domain to the other. Despite many similarities between the two sets of phenomena, there also are important differences (e.g., Meng \& Tong, 2004; Wallis \& Ringelhan, 2013). For example, many binocular rivalry effects can be traced to interocular competition, which clearly is not a factor in the perception of ambiguous stimuli (e.g., Lee \& Blake, 1999; Tong, 2001; Tong \& Engle, 2001). Thus, although data consistent with underlying cross-inhibitory processes in binocular rivalry has been obtained and replicated numerous times with a variety of rivaling stimuli, independent evidence is needed to extend the cross-inhibition hypothesis to ambiguous stimuli. 
Klink, van Ee, and van Wezel (2008b) provided initial evidence in this regard. They studied a bi-stable, rotating, structure-from-motion sphere created from two fields of dots moving from left to right or from right to left, respectively. Observers perceive the direction of sphere rotation to alternate, depending on which field of dots is perceived to be closer. Paralleling binocular rivalry research, Klink et al. (2008a, b) varied the luminance of the dots moving in one direction (e.g., left to right) while holding constant the luminance of the dots moving in the other direction. Their results conformed to Brascamp et al.'s (2006) revised version of Levelt's (1965) Proposition II. Varying the luminance of one set of dots mainly affected the dominance duration of the direction of rotation that was perceived when the stronger set of dots appeared to be closer. Similar asymmetrical findings have been reported by Moreno-Bote, Shpiro, Rinzel, \& Rubin (2010) for ambiguous plaid motion and for gratings depth reversals.

The asymmetrical findings reported by Klink et al. (2008a, b) and Moreno-Bote et al. (2010) are consistent with cross-inhibitory processes affecting the perception of reversible figures. However, the evidence has serious limitations in two respects. First, all of the reversible figures used by Klink et al. and by Moreno-Bote et al. consist of two operationally distinct stimuli, and the ambiguity arises from the way in which the perceptual system integrates these stimuli in the context of motion. For example, the structure-from-motion sphere can be construed as being composed of two transparent surfaces containing dots, and ambiguity in the apparent direction of rotation arises from the perceptual integration of the two surfaces into a single figure when they are superimposed and the dots on each surface move in opposite directions. It is not clear how well findings based on this class of stimuli will generalize to the kind of unified, stationary, two-dimensional stimuli that typically have been used in reversible-figure research. There are both conceptual arguments (Long \& Toppino, 2004) and empirical indications (e.g., Struber\& Stadler, 1999) that different kinds of reversible figures may engage somewhat different processes or the same processes to different degrees, and we will consider some specifically relevant findings a bit later. The second limitation is that the evidence provided by these studies for asymmetrical effects has been obtained with minor variations on a single research strategy. That is, following the lead of binocular-rivalry research, stimulus characteristics (e.g., luminance, contrast) of the two operationally distinct substimuli are manipulated independently (e.g., one is held constant and the other is varied), yielding the critical result that dominance durations are affected primarily for only one perceptual alternative. However, basing theoretical conclusions on one research strategy without converging evidence from other procedures puts one at risk for overestimating the generality and importance of the hypothesized underlying processes.

The primary purpose of the present research is to seek converging evidence for a cross-inhibitory perceptual architecture underlying ambiguous-stimulus perception by using a stationary, two-dimensional stimulus of the sort that has been so prevalent in research with reversible figures and by adopting a complementary research strategy based on the adaptation of one perceptual alternative rather than on variation of the stimulus-input.

We examined the reverse-bias effect using the twodimensional overlapping-squares figure which reverses in perspective so that one can see the figure with its front square oriented down and to the left or up and to the right (see Fig. 2). In a completely within-subjects design, observers participated in a series of trials, each involving an adaptation and test period. During the adaptation period, they viewed one of the unambiguous versions of the figure, a neutral control pattern, or a fixation cross alone. During the subsequent test period, they viewed the standard ambiguous figure and pressed keys to indicate which interpretation they perceived and for how long they perceived it. We reasoned that, if the strength of the stimulus input was held constant while the perceptual system's responsiveness to the input favoring one alternative were weakened through adaptation to a prior unambiguous stimulus, the results should be comparable to those originally obtained by Levelt (1965) in binocular rivalry. That is, the primary effect should be on the dominance durations of the perceptual alternative that was not adapted. Thus, dominance durations for the perceptual alternative that is the opposite of the prior adaptation stimulus should become longer relative to a no adaptation control, whereas dominance durations for the perceptual alternative that is the same as the adaptation stimulus should be relatively unaffected.

In recent work related to our hypothesis, De Jong, Knapen, and van Ee (2012) studied the perception of an ambiguous structure-from-motion stimulus as a function of various preexposure conditions. Among their experiments were two conditions that were similar to ours in that observers engaged in continuous viewing of an unambiguous version of the stimulus before the ambiguous version was presented. They found that, in comparison to a no-pre-exposure baseline, dominance durations of the ambiguous figure were longer for the perceptual alternative to which they had not been pre-exposed but were relatively unchanged for the alternative to which they had been pre-exposed. These findings appear to be consistent with our hypothesis, but other aspects of the results raise questions again about the degree to which findings from this kind of stimulus can be expected to generalize to more common reversible figures such as the stimuli used in our experiment. 


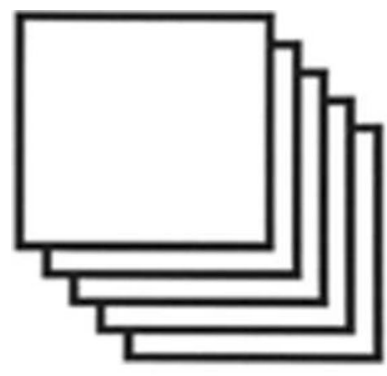

a

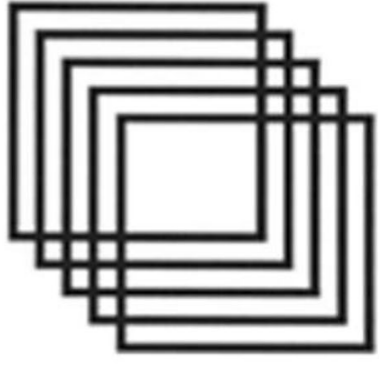

b

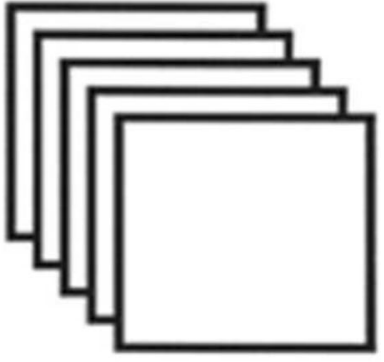

C

Fig. 2 The overlapping-squares ambiguous figure (b), flanked by its unambiguous counterparts: the up-to-the-left orientation (a) and the down-to-theright orientation (c) (Adapted from Long et al, 1992)

Consider, for example, that De Jong et al. (2012) found that the initial percept reported for the ambiguous structure-frommotion stimulus was highly likely to be the same as the unambiguous pre-exposure stimulus that had been presented for an extended period. This result is quite different from the findings obtained in the reverse-bias literature where the interpretation of the initial percept may be the most commonly reported dependent measure. Studies have demonstrated repeatedly with a variety of reversible figures that the initial percept is highly likely to be the opposite of the previously presented unambiguous stimulus (e.g., Carlson, 1953; Emerson, 1979; Harris, 1980; Long \& Moran, 2007; Long \& Olszweski, 1999; Long, et al., 1992, 2002; Petersik, et al., 1984; Virsu, 1975). De Jong et al. also measured the percentage of time that the ambiguous figure was perceived to be the opposite of the unambiguous stimulus, but their results were inconsistent. When the structure-from-motion stimulus was rendered unambiguous by means of binocular disparity, they found that the predominant interpretation of the ambiguous stimulus was the opposite of the unambiguous pre-exposure stimulus, consistent with the results typically obtained in the reverse-bias literature (e.g., Carlson, 1953; Hochberg, 1950; Nawrot \& Blake, 1989; von Grunau, et al., 1984). However, when monocular cues were used to disambiguate the structure-from-motion stimulus (arguably the more relevant condition for generalizing to two-dimensional line-drawing stimuli), the predominance of the interpretation opposite that of the unambiguous stimulus did not seem to differ systematically from the no-pre-exposure baseline. Given that, with some dependent variables, structure-from-motion stimuli yield results that are inconsistently related to the results reported for other reversible figures, de Jong et al.'s findings with dominance durations can be viewed as offering encouragement for our hypothesis, but they certainly are not conclusive.

A secondary goal of the present experiment was to replicate the reverse-bias effect with the dependent measures traditionally used in the literature. We expected: (1) the initial interpretation of the ambiguous stimulus to be the opposite of the adaptation stimulus, (2) the opposite interpretation to be perceived for a greater cumulative amount of time, and (3) the frequency of perceptual reversals to be depressed. Previous experiments have assessed one or two of these effects, but no single study has assessed them all. By demonstrating these effects in the context of a single set of procedures, we hoped to integrate the literature and strengthen the evidence for adaptation by reducing any doubt that these effects are different manifestations of the same underlying processes. By further relating these effects to our findings on dominance durations, we hoped to illuminate the relationship between adaptation and hypothesized cross-inhibitory processes.

\section{Method}

\section{Observers and design}

Observers were 40 Villanova University students who participated for partial fulfillment of the requirements of a general psychology course. They participated in all four conditions of a fully within-subjects design. In every condition, observers engaged in a series of adaptation periods followed by test periods. The adaptation conditions differed with respect to the stimulus to which observers were exposed during the adaptation period: Unambiguous Up Left, Unambiguous Down Right, Control Figure, and Control Fixation Only.

Materials and procedures

Observers were seated with their head restrained by a chin rest, viewing a computer monitor at a distance of $66 \mathrm{~cm}$. Stimuli were black figures, presented on a white background. They consisted of an ambiguous overlapping squares figure (Fig. 2, center panel), an unambiguous version of the figure oriented up and to the left (Fig. 2, left panel), an unambiguous version oriented down and to the right (Fig. 2, right), and two control conditions: a blank white field with a small fixation cross and a figure which consisted of the same number and 
length of black lines as the unambiguous stimuli but which was perceived as a flat (i.e., depthless) unrelated symmetrical pattern. When presented, those stimuli containing any line patterns subtended $4.3^{\circ}$ of visual angle.

Observers participated in 12 experimental trials that were organized into 3 blocks of 4 trials. Within each block, each of the four experimental conditions was presented once, and the order in which they were presented was determined randomly. Just before a trial began, observers were warned to adjust their head in the chin rest and to rest two fingers on the "1" and " 2 " keys of the keyboard's numeric keypad. All trials started with the presentation of a centrally located fixation cross that remained continuously visible throughout the trial. After 1.5 $\mathrm{s}$, the adaptation period began and eventually was followed by the test period.

Depending on the experimental condition, the stimulus observers viewed during the adaptation period was either the unambiguous up-left version of the overlapping squares figure, the unambiguous down-right version of the figure, the neutral control figure consisting of an unrelated pattern of lines, or the fixation cross only (blank field). In the blank field condition, no figure was presented, and the fixation point alone was visible for the duration of the adaptation period. When the unambiguous figures or the control pattern were presented, they were centered on the fixation cross. Observers made no overt responses during the adaptation period. Instructions stressed that their gaze was to remain focused on the fixation cross at all times. The adaptation period lasted for $150 \mathrm{~s}$, after which the computer beeped to announce the beginning of the test phase, and, simultaneously, the ambiguous overlapping squares figure, centered on the fixation cross, replaced the adaptation array. The ambiguous stimulus was presented for $60 \mathrm{~s}$ during which observers were to continue to maintain their focus on the fixation cross. Now, however, they were to report their phenomenal experience by pressing the " 1 " key or the " 2 " key of the keypad to indicate whether they perceived the stimulus to be oriented up and to the left or down and to the right, respectively. In addition, they were instructed to keep the key depressed for as long as they continued to see the figure in that particular orientation. The instructions stressed that the observers should view the figure passively and not do anything that might encourage or discourage reversals from being experienced. Following each test period, observers were allowed to rest and relax before the next experimental trial began. The rest period after most trials lasted $90 \mathrm{~s}$ but was extended to $150 \mathrm{~s}$ after the fourth and eighth trials.

Prior to the experimental trials, observers were shown the stimuli that would be used and received a thorough explanation of the nature of the stimuli and procedures. Then, observers viewed the ambiguous overlapping squares figure for $60 \mathrm{~s}$ and reported their perceptual experiences, using the procedures that would be employed during the test periods of the experimental trials. Finally, observers received four practice trials to familiarize them with the procedures that would be used in the experimental trials. One practice trial corresponded to each of the four experimental conditions and was procedurally identical to the experimental trials for that condition with the exception of a shorter adaptation period of $90 \mathrm{~s}$.

\section{Results}

Our experimental design included two control conditions, one in which observers viewed a pattern of line segments unrelated to the ambiguous overlapping-squares figure during the adaptation period of a trial, and a second in which only the fixation cross was presented during the adaptation period. Preliminary analyses found no reliable differences between these two conditions on any of our dependent variables. Therefore, the data from the two control conditions were combined and treated as a single control for the purposes of our primary analyses.

We first report the results of our experiment with respect to the dependent variables that have been used in the past to study the reverse-bias effect: (1) the total time within the 60 -s test period that observers perceived each interpretation of the ambiguous figure, (2) the proportion of trials on which a particular interpretation was perceived initially (i.e., first percept), and (3) the number of phenomenal reversals experienced during the test period. Then, we turn to the novel analyses involving the duration of the intervals during which each interpretation of the reversible figure was perceptually dominant (i.e., dominance durations). Unless otherwise stated, statistical significance was set at $p=0.05$ for all analyses.

\section{Total time for each alternative}

The dependent variable in these analyses was the total time in the 60 -s test period that observers perceived each interpretation of the reversible overlapping-squares figure. These data were analyzed in a $2 \times 3$ (Perceptual alternative: Up-left vs. Down-right) $\times$ Adaptation condition: Same vs. Opposite vs. Control) repeated-measures ANOVA. That is, the total time of perception was analyzed as a function of the alternative that was being perceived and whether the preceding adaptation period involved an unambiguous stimulus depicting the same orientation, an unambiguous stimulus depicting the opposite interpretation, or a control display. The analysis revealed a significant main effect of adaptation condition, $F(2,78)=$ 35.14, $M S E=71.06$, which can be seen in Fig. 3. As predicted by the adaptation hypothesis, Tukey hsd paired comparisons indicated that all differences were reliable. Thus, compared to the control, the time during which observers perceived a 


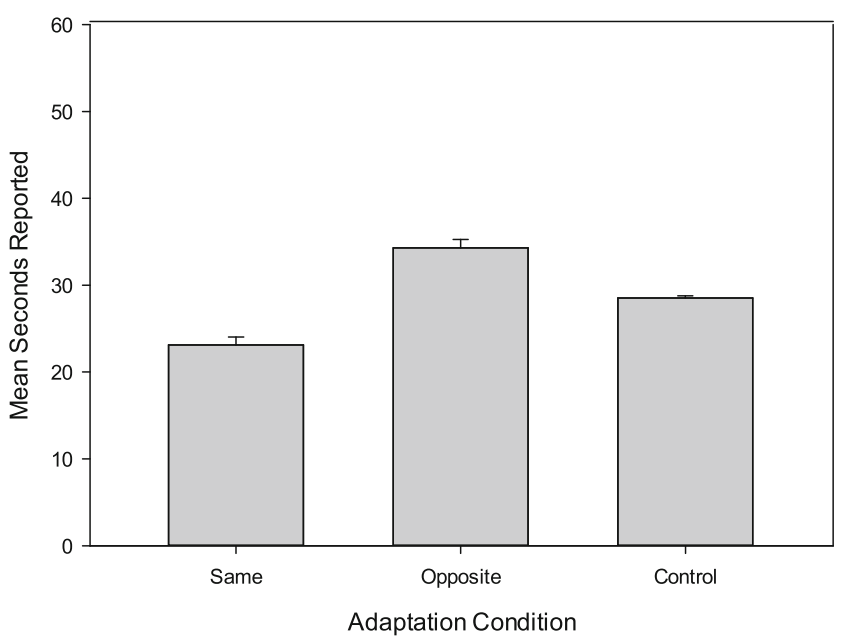

Fig. 3 Mean number of seconds that each alternative was perceived during the 60-s test period when it was the same as, or opposite from, the unambiguous adaptation stimulus and when there was no prior unambiguous stimulus (control). Error bars represent standard error of the mean

particular interpretation was decreased significantly following adaptation to the same interpretation but was increased significantly following adaptation to the opposite interpretation. The ANOVA also revealed a main effect of perceptual alternative, $F(1,39)=71.86, M S E=117.23$, but no reliable interaction, $F(2,78)<1.00$, MSE $=14.66$. The down-right alternative was perceived for a greater share of the time $(M=$ $35.58 \mathrm{~s})$ than the up-left orientation $(M=22.73 \mathrm{~s})$. This reflects a common bias, also seen with the Necker cube, to perceive a stimulus as though it were being viewed from above (see Long \& Batterman, 2012, for a recent discussion of the problems resulting from pre-existing biases evident with many reversible figures). This bias, however, was orthogonal to the effects of adaptation as revealed by the fact that the interaction failed to approach significance.

\section{Initial perception (first percept)}

The left and middle panels of Fig. 4 show, respectively, the mean proportion of trials on which observers reported initially perceiving the up-left and the down-right interpretation of the reversible figure as a function of whether adaptation involved exposure to the same interpretation, the opposite interpretation, or neither (control condition). One-way, repeated-measures ANOVAs on the data in each panel revealed significant effects of adaptation condition, $F_{\mathrm{S}}(2,78)=42.78, M S E=.084$. The most important aspect of the findings is that observers were much more likely to perceive a given interpretation of the reversible figure if they had been adapted to the opposite interpretation rather than the same interpretation (see the left and center bars of the left and middle panels). This difference was significant according to Tukey hsd paired comparisons, regardless of the perceptual alternative being reported.
Comparisons involving the control condition were more complicated. As expected, the proportion of both percepts was highest following opposite adaptation and lowest after same adaptation, with control adaptation falling in between. If the data are collapsed over the two alternatives, a rather straightforward picture emerges. As shown in the right panel of Fig. 4, there clearly was a reduced proportion of reports in the test period for the same alternative and a greater percentage of reports for the opposite alternative. However, for reports of the up-left percept (left panel), a reliable difference was obtained between the control- and opposite-adaptation conditions, whereas, for reports of the down-right percept (right panel), a reliable difference was found between the control- and sameadaptation conditions. Neither the reduction in up-left percepts produced by the same-adaptation condition nor the increase in down-right percepts produced by the oppositeadaptation condition attained statistical significance relative to the controls. These failures seem to be a consequence of the overall bias in favor of perceiving the down-right orientation that was observed in the previous analysis of total-time data. With regard to the initial-percept data, the bias is most apparent in the control-condition (see the rightmost bar in left and middle panels of Fig. 4). Observers initially perceived the down-right alternative on $86 \%$ of the trials and the up-left alternative on only $14 \%$. This high proportion of down-right percepts and correspondingly low proportion of up-left percepts suggest ceiling and floor effects, respectively, constraining the size of the differences that can be obtained. Thus, there is little room for a decrease in the perception of the up-left alternative following adaptation to an unambiguous stimulus depicting the same interpretation, and little room for an increase in the perception of the down-right alternative after adaptation to an unambiguous stimulus showing the opposite interpretation.

\section{Number of reversals}

The adaptation hypothesis predicts that the number of reversals perceived during the test period while viewing an ambiguous figure (i.e., the reversal rate) should be suppressed following adaptation to an unambiguous version of the figure because the sensitivity of the neural network underlying one of the interpretations has been reduced so it cannot compete effectively and the alternate interpretation dominates. Furthermore, the reversal rate should be lowest at the outset of the test period and should increase over time as the adapted perceptual alternative undergoes recovery. Therefore, we conducted a $3 \times 4$ repeated-measures ANOVA on the number of reported reversals as a function of adaptation (Up Left vs. Down Right vs. Control) and four successive 15-s intervals within the test period. Results indicated main effects of Adaptation, $F(2,78)=17.77, M S E=1.65$, and of Interval, $F(3,117)=9.06, M S E=0.68$, but these effects were qualified 


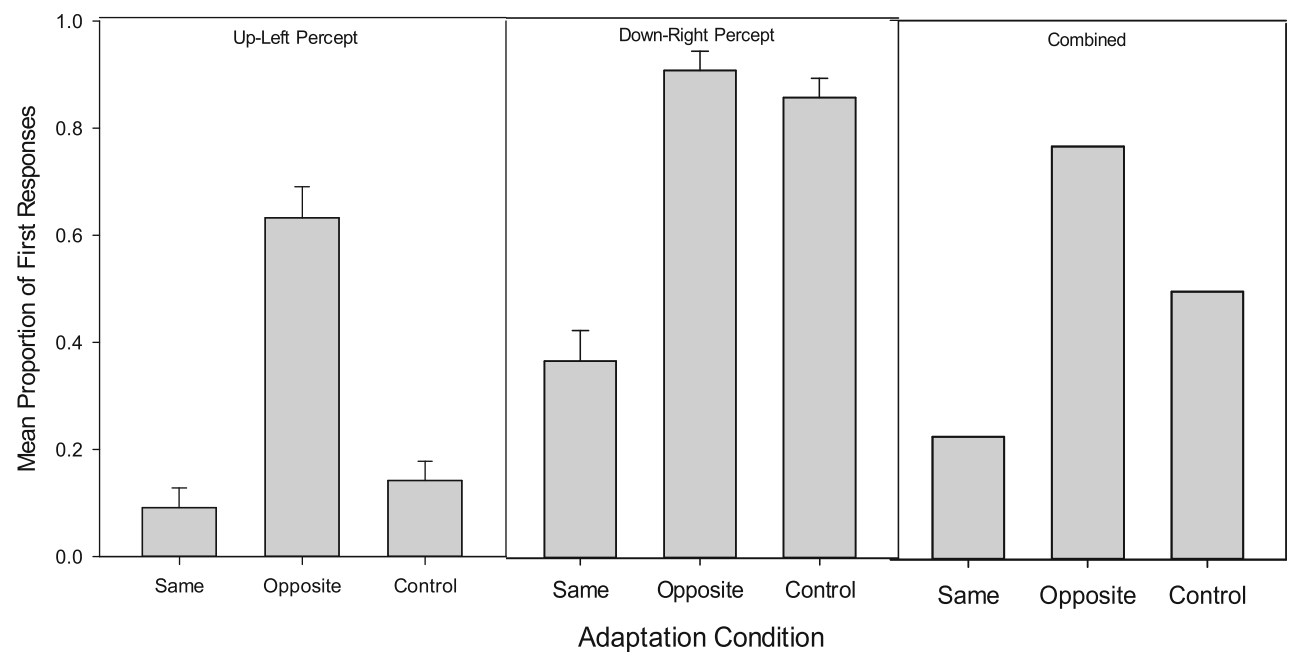

Fig. 4 Mean proportion of trials on which the up-left and down-right percepts (left and middle panels, respectively) were reported to have been perceived first as a function of adaptation condition (same, opposite, or control). The right panel presents the data for both percepts combined. Error bars represent standard error of the mean

interval, the number of reversals no longer differed significantly between either adaptation condition and the control, $t$ 's $(39) \leq 1.55$.

\section{Duration of periods of dominance}

A major purpose of the present experiment was to extend research on the reverse bias effect by investigating how adaptation to an unambiguous version of a reversible figure affects the duration of periods of dominance for the two alternatives when observers subsequently view the ambiguous stimulus. To achieve this goal, we needed a sample of observers who provided a minimally sufficient number of discrete periods of dominance. Thus, we included observers in these analyses only if their data yielded at least six periods of dominance in each adaptation condition (i.e., the observer reported each of the two percepts at least three times). Because a number of observers perceived few reversals during some test periods, this restriction reduced our sample from 40 to 28 observers. The elimination of so many observers concerned us for fear that the new sample was somehow atypical and not representative of the sample as a whole. Therefore, we re-conducted all of the previously reported analyses with the new, smaller sample. The outcome of all of the analyses was the same for the 28-observer sample as it had been for the full 40-observer sample. Thus, the elimination of the observers who exhibited the slowest rates of reversal did not seem to otherwise bias the remaining sample.

Mean dominance duration for the entire test period. The mean dominance duration for each perceptual alternative across the entire test period was determined for each observer and each adaptation condition. These data were submitted to a $2 \times 3$ (Perceptual alternative $\times$ Adaptation condition) repeated-measures ANOVA. The most important result was
Fig. 5 Mean number of reversals during the 60-s test period as a function of successive 15-s intervals and adaptation condition. Error bars represent standard error of the mean 
a significant main effect of adaptation condition, $F(2,54)=$ 13.91, $M S E=5.92$ (see Fig. 6). Post-hoc Tukey hsd paired comparisons confirmed what is apparent in the figure. Relative to the control condition, adapting to an unambiguous version of a reversible figure significantly increased the mean dominance duration of the opposite percept but had no effect on the mean duration of the same percept when the ambiguous version of the figure was subsequently viewed. The ANOVA also revealed that the mean dominance duration generally was greater for the down-right than for the up-left orientation, $F(1$, 27) $=11.55, M S E=7.46, M \mathrm{~s}=5.61 \mathrm{~s}$ and $4.18 \mathrm{~s}$, respectively. This finding appears to be another reflection of the general bias to perceive the down-right interpretation of the overlapping-squares figure. However, the lack of a significant interaction, $F(2,54)=1.95, M S E=2.76$, indicates that this bias did not influence the critical adaptation effect.

Duration of the first three periods of dominance for each percept

In order to gain a clearer understanding of how adaptation affects dominance duration, we analyzed the duration of the first three periods of dominance for each percept (up left and down right) in the test period. On the basis of the adaptation hypothesis, we expected that, following adaptation to an unambiguous stimulus, the mean dominance duration would be greater for the opposite than for the same perceptual interpretation of the ambiguous stimulus. We further expected that this difference would be greatest for the first period of dominance and would diminish over successive periods of dominance. An important open question concerned how the dominance durations in the control condition would compare to those in the same and opposite adaptation conditions.

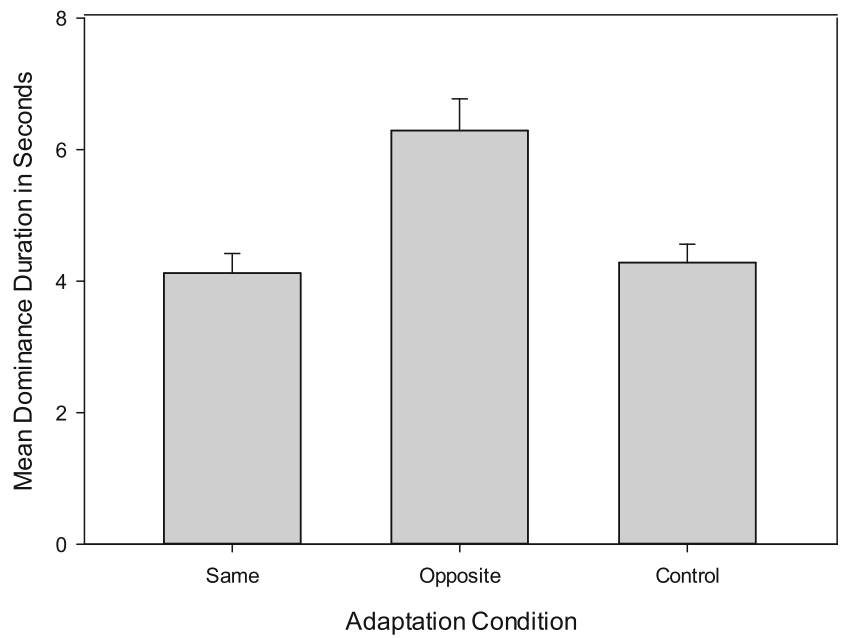

Fig. 6 Mean dominance duration in seconds during the 60-s test period as a function of adaptation condition. Error bars represent standard error of the mean
Mean dominance duration was submitted to a $3 \times 2 \times 3$ repeated-measures ANOVA involving adaptation condition (same vs. opposite vs. control), perceptual alternative (up left vs. down right), and successive periods of dominance (first vs. second vs. third). We found a significant main effect of adaptation condition, $F(2,54)=30.27, M S E=9.90$. Tukey hsd post hoc comparisons indicated that, in comparison to the control condition, adaptation increased the dominance duration of the opposite percept but not of the same percept. The ANOVA also revealed a main effect of perceptual alternative, $F(1,27)=$ $21.30, M S E=18.27$, with the down-right percept $(M=5.81 \mathrm{~s})$ enjoying longer periods of dominance than the up-left percept $(M=4.06 \mathrm{~s})$. There also was an adaptation condition $\times$ perceptual alternative interaction, $F(2,54)=7.42, M S E=10.65$, such that the general difference in dominance duration favoring the down-right over the up-left interpretation was even greater in the opposite adaptation condition. Finally, and most importantly, there was a significant interaction, $F(4,108)=6.51, M S E=$ 4.91 , between adaptation condition and successive periods of dominance (see Fig. 7), whereas no other effect, including the three-way interaction, approached significance, all $F_{\mathrm{S}}<1.56$.

Inspection of Fig. 7 shows the same pattern among adaptation conditions as was evident in the main effect of that variable, with the obtained differences being greatest for the first period of dominance and then diminishing over succeeding dominance periods. This description of the effect was borne out when the interaction was probed. Simple effects of adaptation condition at each dominance period indicated a significant adaptation effect in all three dominance periods, $F_{\mathrm{S}}(2,108)=$ $39.52,9.65$, and 4.84 , for the first, second, and third dominance periods, respectively, MSE $=6.73$. Follow-up Tukey hsd paired comparisons indicated that, in the first and second dominance period, adaptation to an unambiguous stimulus resulted in a longer period of dominance for the opposite percept than for the control condition, whereas the duration of the same percept

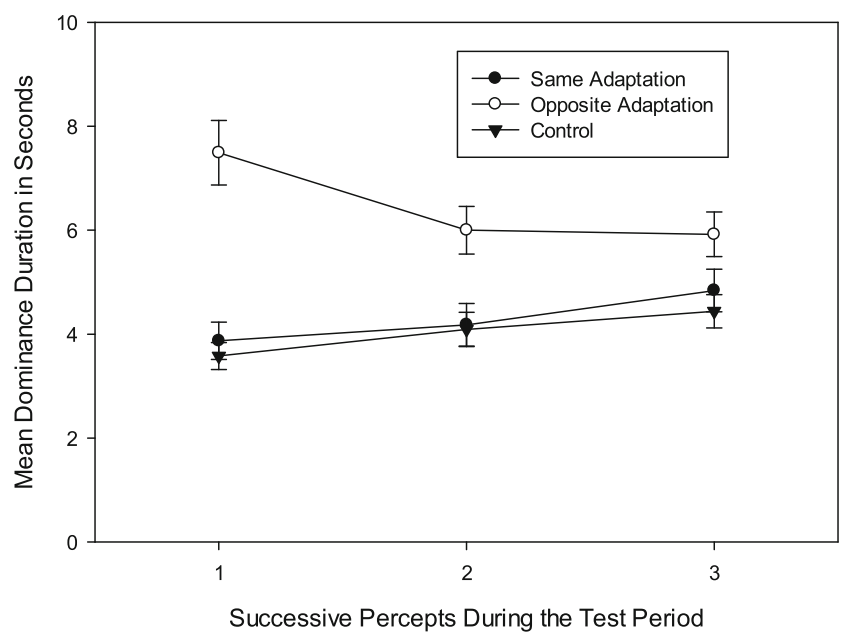

Fig. 7 Mean dominance duration of the first three percepts reported during the test period as a function of adaptation condition. Error bars represent standard error of the mean 


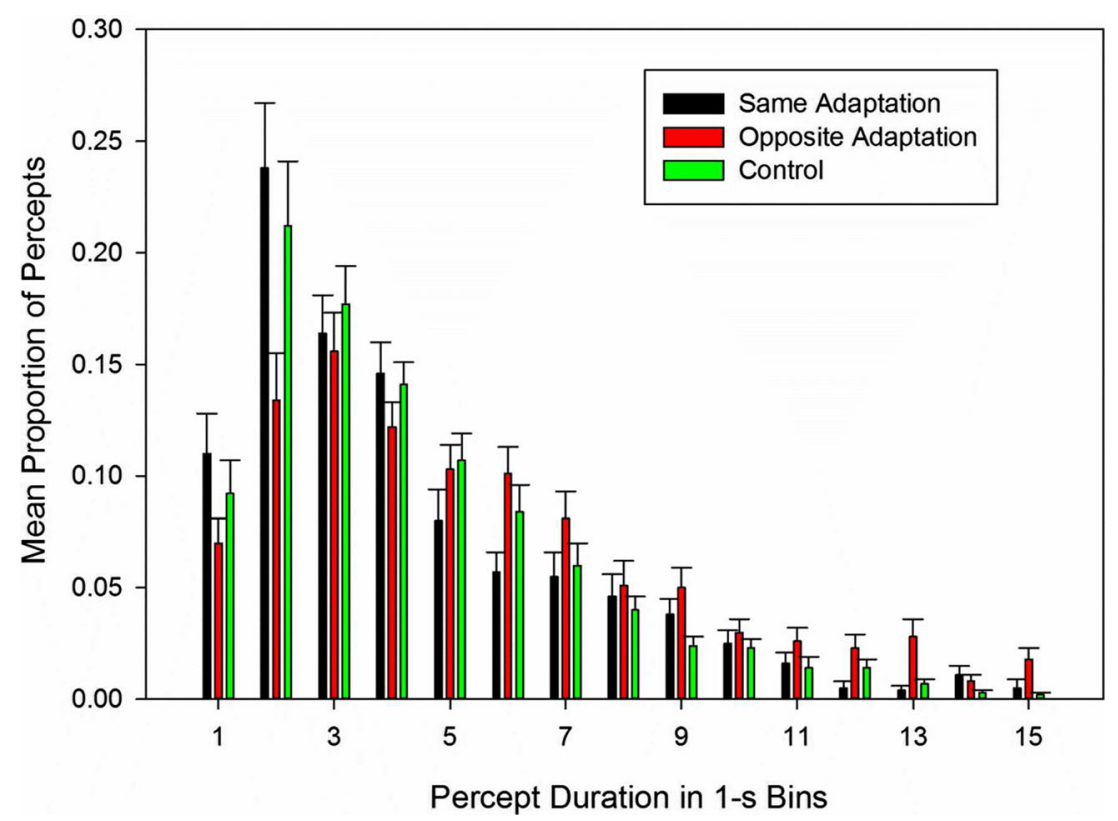

Fig. 8 Mean proportion of percepts as a function of 1-s duration intervals (or bins) and whether the percept was preceded by an adaptation stimulus that was the same (Same Adaptation), an adaptation stimulus that was the

was unchanged relative to the control. Although the pattern of results was the same in the third dominance period, the Tukey hsd comparisons failed to reach significance. In view of the fact that the simple effect of adaptation had been significant, we felt that a less conservative, more powerful test was appropriate. Therefore, for the third period of dominance, we used $t$-tests with a Bonferronni correction to compare the control condition with the same and opposite adaptation conditions. Once again, compared to the control condition, adaptation to an unambiguous stimulus led to longer dominance durations for the opposite percept, $t(27)=3.73$, but had no effect on the durations for the same percept, $t(27)=1.12$.

\section{Distribution of dominance durations}

Figure 8 depicts the mean proportion of percepts as a function of prior adaptation history and the length of the percept's duration, measured in 1-s intervals. That is, interval 1 is for percept durations of $1 \mathrm{~s}$ and less. Interval 2 is for percept durations greater than $1 \mathrm{~s}$ up to $2 \mathrm{~s}$, and so forth. It is apparent from the figure that the distributions are unimodal and skewed to the right. This is nicely consistent with previous research on the distribution of dominance durations (e.g., Borsellino et al., 1972; Brascamp et al., 2005). However, unlike prior research, we did not intend, nor did we design our research, to determine the theoretical form of the distribution (i.e., whether the distribution would be best described as a gamma distribution or something else). Our primary interest lay in how the empirical distributions might be altered as a function of prior adaptation in the reverse-bias paradigm. opposite (Opposite Adaptation), or no adaptation stimulus (Control). The maximum interval of $15 \mathrm{~s}$ was an arbitrary choice. Error bars represent standard error of the mean

Figure 8 indicates that the distribution of percepts that are the same as a prior adaptation stimulus is similar to that for percepts when there was no prior adaptation (control); that is, both distributions rise from the 1-s bin to the 2-s bin and then trail off across successive bins in a nearly identical fashion. In contrast, when percepts that are the opposite of a prior adaptation stimulus are considered, the distribution is flattened and the mode is shifted to the right, toward longer durations. Although these overall trends are readily apparent in Fig. 8, it also is apparent that there is considerable variability from one 1-s bin to the next. Therefore, to stabilize the data for analysis, we combined durations into 3 -s bins. Thus, the first bin represented percepts with durations of $3 \mathrm{~s}$ or less. The second bin represented percepts with durations longer than $3 \mathrm{~s}$ but not more than $6 \mathrm{~s}$, and so forth until the final bin which represented percepts with durations greater than $12 \mathrm{~s}$ up to 15 s. We submitted these data to a 3 X2X5 repeated-measures ANOVA involving prior adaptation history (same as a prior adaption stimulus vs. opposite from a prior adaptation stimulus vs. control with no prior adaptation), perceptual alternative (up left vs. down right), and duration (bins 1-3 s through 13$15 \mathrm{~s})$. There were three reliable effects: the main effect of duration, $F(4,108)=59.96, M S E=.09$, the interaction of perceptual alternative $\mathrm{X}$ duration, $F(4,108)=14.18, M S E=$ .013 , and the interaction of prior adaptation history $\mathrm{X}$ duration, $F(8,216)=6.12, M S E=.02 .^{2}$

The interaction of perceptual alternative and duration was probed by examining the simple effects of perceptual

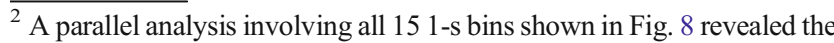
same overall effects.
} 
alternative at each level of duration. The results were consistent with, and expanded upon, previous analyses showing a bias to perceive the reversible figure in the down-right orientation. In this case, the mean proportion of percepts at the shortest durations (bin 1-3 s) was greater for the up-left than for the down-right interpretation, $F(1,110)=43.0, M S E=.013$, whereas the mean proportion of percepts at the longer durations was greater for the down-right than for the up-left percept, with the difference being significant for the 7-9 s and 10-12 s bins, $F \mathrm{~s}(1,110)=4.39$ and 5.77 , respectively, and marginally significant for the $13-15 \mathrm{~s}$ bin, $F(1,110)=3.85$, $P=.052$, all MSE $=.013$.

The prior adaptation history $\mathrm{X}$ duration interaction seems to reflect the fact that, at the shortest durations, the percept that was the opposite of the adaptation stimulus occurred less often than either of the other percept types, whereas, for longer durations, it consistently occurred more often than the other types of percept. This was borne out when we probed the interaction by analyzing the simple effects of prior adaptation history for each duration, followed by paired comparisons when appropriate. The simple effect of adaptation history was significant for the 1-3 s duration bin, $F(2,220)=$ $18.63, M S E=.019$. Tukey hsd paired comparisons indicated that the proportion of percepts that were the opposite of the prior adaptation stimulus was lower than the proportion of percepts that were the same as the prior adaptation stimulus and the proportion of percepts with no prior adaptation history (control). There was no difference in the proportion with which the latter types of percept occurred. In the 4-6 s range, the proportion of percepts did not differ significantly as a function of prior adaptation history, $F(2,220)=2.00, M S E$ $=.019$. However, in the 7-9 $\mathrm{s}$ duration range, the proportion of opposite adaptation percepts $(M=.181)$ was greater than the proportion of control- and same-adaptation percepts $(M \mathrm{~s}=$ .124 and .139 , respectively), $F(2,220)=2.63, M S E=.019, P$ $=.074$. Although conservative Tukey post-hoc tests did not reveal any significant differences among the adaptation history conditions at this duration, more powerful $t$ tests with a Bonferroni correction indicated that the proportion of opposite-adaptation percepts $(M=.181)$ was reliably greater than the proportion of no-adaptation (control) percepts $(M=$ $.124), t(27)=2.50$, which, in turn, did not differ significantly from the proportion of same-adaptation percepts $(M=.139), t$ $(27)=1.28$. The same pattern among adaptation history conditions was obtained in the two longest duration ranges although the effects were not statistically reliable, $F$ s $(2,220) \leq 1.00, M S E=.019$.

Finally, we sought statistical support for the apparent peak shift (deflection of the mode toward a longer duration) that was apparent for percepts that were the opposite of the prior adaptation stimulus. We determined each observer's modal duration for each combination of perceptual alternative (up left and down right) and prior adaptation history condition (same, opposite, and control). ${ }^{3}$ These data were submitted to a $2 \times 3$ (perceptual alternative $\times$ adaptation history condition) repeated measures ANOVA. The results indicated that the mean modal duration was longer for the down-right than for the up-left alternative, $F(1,27)=6.18, M S E=3.70$, reflecting once again the bias in favor of perceiving the down-right interpretation. More importantly, modal durations differed as a function of the adaptation history condition, $F(2,54)=6.39$, $M S E=2.96$. Tukey hsd paired comparisons indicated that the modal duration was longer for percepts that were the opposite of the adaptation stimulus ( $\mathrm{M}=3.53 \mathrm{~s})$ than for percepts that were the same as the prior adaptation stimulus $(M=2.42 \mathrm{~s})$ and for percepts with no prior adaptation $(M=2.69 \mathrm{~s})$. The modal duration of the latter types of percept did not differ significantly from one another.

\section{Discussion}

The present results advance our understanding of the reversebias effect empirically by affording the first integrated description of how adaptation to an unambiguous stimulus affects the perception of the subsequent reversible figure. More importantly, the findings strengthen existing empirical support for the involvement of adaptation processes in the perception of reversible figures and, in particular, offer insight into the inhibitory architecture of the processing system within which adaptation contributes to perceptual bistability in these stimuli.

An integrated empirical description of the reverse-bias effect emerged from our use of multiple measures of the phenomenon in the context of a single set of experimental procedures. In particular, we extended previous research on the reverse-bias effect by investigating how adaptation to an unambiguous version of a reversible figure affects the dominance duration of the alternative interpretations of the subsequently presented ambiguous stimulus. We found that adaptation to the unambiguous stimulus significantly increases the average dominance duration of the opposite interpretation of the ambiguous stimulus while having little effect on the dominance duration of the same interpretation. In other words, the opposite alternative is perceived for a longer period of time when it is dominant. This, of course, results in the alternative that was adapted (same percept) being suppressed for an equivalently longer period of time. When the alternative that is the same as the adaptation stimulus is dominant in the test

\footnotetext{
${ }^{3}$ If an observer produced multiple modes in a given condition such that two or more durations were tied for the highest number of percepts, we entered as the mode for that observer and condition the mean of his or her modal durations.
} 
period, however, it shows no effect of prior adaptation. Its duration of dominance is equally long regardless of whether it is perceived after exposure to an adapting stimulus or not. A similar result was obtained by de Jong et al. (2012) with a rotating, structure-from-motion sphere. However, as we discussed earlier, the generality of their findings was suspect because other aspects of their results were inconsistently related to established results in the reverse-bias literature.

In contrast, we replicated all three major patterns of findings that were offered previously as evidence of the reversebias effect, and the addition of the dominance-duration data complemented and clarified the nature of these results. Consistent with previous research (e.g., Hochberg, 1950; von Grunau et al., 1984), we found that, relative to the control condition, adaptation to an unambiguous version of the ambiguous stimulus led to the opposite interpretation being perceived for a greater proportion of the post adaptation test period and to the same interpretation being perceived for a lesser proportion of the test period. Our results extend previous findings by showing that this effect can be attributed to longer dominance durations for the opposite interpretation but not to shorter dominance durations for the same interpretation. Again, in agreement with previous research (e.g, Long \& Moran, 2007; Long \& Batterman, 2012; Long et al, 1992; Petersik et al., 1984; Virsu, 1975), we found that the opposite alternative was more likely to be perceived initially than the same alternative when the reversible figure was first presented after the adaptation period. Our results elaborate on this finding by indicating that the duration of the first perception of the opposite interpretation tends to be longer than it would have been without prior adaptation, whereas the duration of the first perception of the same interpretation is not affected. Finally, like a number of previous studies (e.g., Long \& Batterman, 2012; Long \& Moran, 2007; Long et al., 1992; Long \& Olszweski, 1999), we found that the frequency of reversals was initially suppressed following adaptation to an unambiguous stimulus but recovered during the course of the test period. Our findings also indicate that the reduced reversal rate can be attributed to longer dominance durations of the opposite percept and that the reversal rate increases during recovery as the dominance durations of the opposite interpretation become shorter with the passage of time, approaching the level produced by the control condition.

At the theoretical level, our results provide strong support for the involvement of time-dependent neural adaptation processes in the perception of reversible figures. Specifically, the perception of either alternative of a reversible figure can be controlled experimentally by means of extended exposure to one of the unambiguous versions of the stimulus. When the ambiguous stimulus is presented subsequently, the figural interpretation for the same version as viewed during the adaptation period loses the competition with the opposite alternative which then enjoys a lengthened period of dominance.
Although our results clearly support a role for adaptation in the processes underlying figure reversals, they are compatible with the contribution of a stochastic process as well. Determining precisely how the adaptation and stochastic processes are coordinated, however, is beyond the scope of the current research. Several options may be possible, but the simplest, most parsimonious hypothesis may be that adaptation of alternative pools of neurons determines which competing perceptual alternative gains dominance, and that the process of neural adaptation and/or the process of recovery from adaptation have a strongly stochastic character, presumably reflecting neural noise.

As we noted in the Introduction, adaptation alone (with or without stochastic properties) cannot account for perceptual bistability. Adaptation addresses how the relative strength of perceptual alternatives of a reversible figure may wax and wane over the course of an extended viewing period, but it does not explain why the perception of the stronger alternative precludes the perception of the weaker one. This is best understood in terms of the architecture of the processing system underlying bistable perception. It appears that the two perceptual alternatives reciprocally inhibit one another, such that, when one alternative is dominant, the other is suppressed and vice versa.

Various architectures can be envisioned. For example, the simplest might be one in which the neural structures underlying the alternative interpretations of an ambiguous stimulus compete for a single, common processing channel or limitedcapacity resource. In this selective-attention-like view, a single mechanism accounts simultaneously for the dominance of one interpretation and the suppression of the other (e.g., Fox \& Rasche, 1969). Thus, anything (including adaptation) that affects the dominance of one perceptual representation should have an equivalent and opposite effect on the other representation. But such an architecture is inconsistent with findings like ours. The asymmetry of our results suggests a crossinhibitory architecture in which the two perceptual representations associated with a bistable ambiguous stimulus inhibit one another via separate pathways that operate at least somewhat independently. Thus, adapting one percept (P1) may increase the time that it can be inhibited or suppressed by the competing percept (P2) without necessarily affecting the time that P2 can be inhibited by P1 when the latter finally becomes dominant.

Asymmetrical results suggestive of a cross-inhibitory architecture have been obtained in previous research involving different perceptual phenomena as a consequence of varying the strength of the stimulus input favoring one perceptual alternative while holding constant the input strength related to the competing alternative. Thus, in binocular rivalry experiments, when the strength of the stimulus input is varied for one eye and is fixed for the other, dominance durations are affected primarily for a single eye (e.g., Levelt, 1965; 
Brascamp et al., 2006). This pattern of results has been extended to one class of ambiguous figures consisting of two operationally independent stimuli which become perceptually integrated into a single figure in the context of motion (e.g., Klink et al., 2008a, b; Moreno-Bote et al., 2010). Varying the stimulus characteristics of one of the component stimuli (e.g., the luminance of dots moving in one direction within a spherical structure-from-motion display) primarily affects the dominance durations of only one of the perceptual alternatives.

Our results complement and extend these previous findings in two ways. Most importantly, we obtained the same critical pattern of results, suggestive of cross-inhibition, without varying the strength of the stimulus input. Our strategy was to hold the stimulus characteristics of the ambiguous figure constant while using prior adaptation to vary the responsiveness of the perceptual system to one perceptual alternative or the other. Our procedures are operationally different but conceptually parallel to those underlying Levelt's (1965) original Proposition II. In the classic binocular rivalry experiments, the strength of input to one eye was varied down from a level that remained fixed for the other eye. In our experiment, the responsiveness of the system to input favoring one perceptual interpretation was lowered via adaptation. In both lines of research, the dominance durations were changed (i.e., became longer) primarily for the perceptual interpretation that had not been varied by means of input strength or adaptation. Our results, however, make clear that this critical finding is not simply a stimulus effect. Rather, it is a result of settings within the perceptual system that may be influenced by a variety of factors, including stimulus input and prior experience.

Another way in which our research complements and extends previous work is by obtaining evidence consistent with cross-inhibitory processes in a stationary, two-dimensional ambiguous stimulus of the sort that has dominated the reversiblefigure literature for over a century and a half (Long \& Toppino, 2004). Klink et al. (2008a, b) and Moreno-Bote et al. (2010) obtained evidence consistent with a cross-inhibitory architecture underlying the perception of a different class of ambiguous stimulus (e.g., a spherical structure-from-motion display), and de Jong et al. (2012) obtained suggestive results using the same kind of stimulus but experimental procedures similar to those used here. Our findings provide a critical link in establishing a pattern of results that is consistent with a cross-inhibitory perceptual architecture and that extends to bistable perception in a variety of contexts, including binocular rivalry and very different types of ambiguous figures.

Finally, nothing in our methodology allows us to identify the adaptation effects we obtained as manifestations of bottom-up or top-down processes. It is possible that adaptation effects can occur at either or both levels. Nevertheless, we lean toward a bottom-up interpretation on the basis of research indicating that the reverse bias effect is contingent on the unambiguous adaptation figure and the ambiguous test figure stimulating the same retinal and cortical tissue. Hochberg (1950) obtained the reverse-bias effect with a Maltese cross when the adaptation and test figures appeared in the same orientation (with corresponding stimulus elements projected to the same spatial location) but not when they were rotated with respect to one another. Von Grunau et al. (1984), working with the overlapping squares figure, found that when the retinal distance between the adaptation and test stimuli varied by as little as $1.5^{\circ}$, the reverse bias effect was largely eliminated. Similarly, Long and Moran (2007) found that the reverse-bias effect was eliminated when unambiguous and ambiguous rotating cubes were of sufficiently different sizes $\left(2^{\circ}\right.$ vs. $\left.6^{\circ}\right)$, thus, presumably stimulating different neural channels. These findings seem to implicate bottom-up processes because retinal localization of process is characteristic of early stages of visual processing. However, we are not suggesting that the competing perceptual representations are low-level structures. They almost surely are not. Nevertheless, high level perceptual representations may be critically supported by lower level structures that are susceptible to adaptation.

\section{References}

Attneave, F. (1971). Multistability in perception. Scientific American, $225,62-71$.

Babich, S., \& Standing, L. (1981). Satiation effects with reversible figures. Perceptual and Motor Skills, 52, 203-210.

Blake, R., Sobel, K. V., \& Gilroy, L. A. (2003). Visual motion retards alternations between conflicting perceptual interpretations. Neuron, 39, 869-878.

Borsellino, A., de Marco, A., Alazetta, A., Rinesi, S., \& Bartolini, R. (1972). Reversal time distribution in the perception of visual ambiguous stimuli. Kybernetik, 10, 139-144.

Bossink, C. J. H., Stalmeier, P. F. M., \& de Weert, C. M. M. (1993). A test of Levelt's second proposition for binocular rivalry. Vision Research, 33, 1413-1419.

Brascamp, J. W., van Ee, R., Pestman, W. R., \& van den Berg, A. V. (2005). Distributions of alternation rates in various forms of bistable perception. Journal of Vision, 5, 287-298.

Brascamp, J. W., van Ee, R., Noest, A. J., Jacobs, R. H., \& van den Berg, A. V. (2006). The time course of binocular rivalry reveals a fundamental role of noise. Journal of Vision, 6, 1244-1256.

Bugelski, B. R., \& Alampay, D. A. (1961). The role of frequency in developing perceptual sets. Canadian Journal of Psychology, 15, 205-211.

Carlson, V. R. (1953). Satiation in a reversible perspective figure. Journal of Experimental Psychology, 45, 442-448.

de Jong, M. C., Knapen, T., \& van Ee, R. (2012). Opposite influence of perceptual memory on initial and prolonged perception of sensory ambiguity. PLoS One, 7(1), e30595.

Dornic, S. (1967). Measurement of satiation in reversible figures. Studia Psychologica, 9, 18-24.

Emerson, P. L. (1979). Necker cube: Duration of preexposure of an unambiguous form. Bulletin of the Psychonomic Society, 14, $397-$ 400 . 
Fisher, G. H. (1967). Preparation of ambiguous stimulus materials. Perception \& Psychophysics, 2, 421-422.

Flugel, J. C. (1913). The influence of attention in illusions of reversible perspective. British Journal of Psychology, 5, 357-397.

Fox, R., \& Herrmann, J. (1967). Stochastic properties of binocular rivalry alternations. Perception \& Psychophysics, 2, 432-446.

Fox, R., \& Rasche, F. (1969). Binocular rivalry and reciprocal inhibition. Perception \& Psychophysics, 5, 215-217.

Girgus, J. J., Rock, I., \& Egatz, R. (1977). The effects of knowledge of reversibility on the reversibility of ambiguous figures. Perception \& Psychophysics, 22, 550-556.

Harris, J. P. (1980). How does adaptation to disparity affect the perception of reversible figures. American Journal of Psychology, 93, 445-457.

Hochberg, J. (1950). Figure-ground reversal as a function of visual satiation. Journal of Experimental Psychology, 40, 682-688.

Hochberg, J., \& Peterson, M. A. (1987). Piecemeal organization and cognitive components in object perception: Perceptually coupled responses to moving objects. Journal of Experimental Psychology: General, 116, 370-380.

Kang, M., \& Blake, R. (2010). What causes alternations in dominance during binocular rivalry? Attention, Perception, \& Psychophysics, 72, 179-186.

Klink, P. C., Van Ee, R., Nijs, M. M., Brouwer, G. J., Noest, A. J., \& Van Wezel, R. J. A. (2008a). Early interactions between neuronal adaptation and voluntary control determine perceptual choices in bistable vision. Journal of Vision, 8(5), 16.1-18.

Klink, P. C., van Ee, R., \& van Wezel, R. J. A. (2008b). General validity of Levelt's propositions reveals common computational mechanisms for visual rivalry. PLoS ONE, 3(10), e3473.

Kohler, W. (1940). Dynamics in psychology. New York: Liveright.

Kohler, W., \& Wallach, H. (1944). Figural aftereffects: An investigation of visual processes. Proceedings of the American Philosophical Association, 88, 269-357.

Kornmeier, J., \& Bach, M. (2004). Early neural activity in Necker cube reversal: Evidence for low-level processing of a gestalt phenomenon. Psychophysiology, 41, 1-8.

Kornmeier, J., \& Bach, M. (2012). Ambiguous figures: What happens in the brain when perception changes but not the stimulus. Frontiers in Human Neuroscience, 6(51), 1-23.

Kornmeier, J., Pfaffle, M. \& Bach, M. (2011). Necker cube: Stimulusrelated (low-level) and percept-related (high-level) EEG signatures early in occipital cortex. Journal of Vision, 11(9): 12, 1-11.

Laing, C. R., \& Chow, C. C. (2002). A spiking neuron model for binocular rivalry. Journal of Computational Neuroscience, 12, 3953.

Lee, S., \& Blake, R. (1999). Rival ideas about binocular rivalry. Vision Research, 39, 1447-1454.

Leeper, R. (1935). A study of a neglected portion of the field of learning: The development of sensory organization. Journal of Genetic Psychology, 46, 41-75.

Lehky, S. R. (1988). An astable multivibrator model of binocular rivalry. Perception, 17, 215-228.

Leopold, D. A., \& Logothetis, N. K. (1999). Multistable phenomena: changing views in perception. Trends in Cognitive Sciences, 3, 254 264.

Levelt, W. J. M. (1965). On binocular rivalry. Soesterberg, The Netherlands: Institute for Perception R.V.O.-T.N.O.

Long, G. M., \& Batterman, J. M. (2012). Dissecting perceptual processes with a new tri-stable reversible figure. Perception, 41, 1163-1185.

Long, G. M., \& Moran, C. J. (2007). How to keep a reversible figure from reversing: Teasing out top-down and bottom-up processes. Perception, 36, 431-445.

Long, G. M., \& Olszewski, A. D. (1999). To reverse or not to reverse: When is an ambiguous figure not ambiguous? American Journal of Psychology, 112, 41-71.
Long, G. M., \& Toppino, T. C. (1981). Multiple representations of the same reversible figure: Implications for cognitive decisional interpretations. Perception, 10, 231-234.

Long, G. M., \& Toppino, T. C. (2004). Enduring interest in perceptual ambiguity: Alternating views in reversible figures. Psychological Bulletin, 130, 748-768.

Long, G. M., Toppino, T. C., \& Kostenbauder, J. F. (1983). As the cube turns: Evidence for two processes in the perception of a dynamic reversible figure. Perception \& Psychophysics, 34, 29-38.

Long, G. M., Toppino, T. C., \& Mondin, G. W. (1992). Prime time: Fatigue and set effects in the perception of reversible figures. Perception \& Psychophysics, 52, 609-616.

Long, G. M., Stewart, J. A., \& Glancey, D. (2002). Configural biases and reversible figures: Evidence of multi-level grouping effects. American Journal of Psychology, 115, 581-607.

Lynn, R. (1961). Reversible perspective as a function of stimulus-intensity. American Journal of Psychology, 74, 131-133.

Meng, M., \& Tong, F. (2004). Can attention selectively bias bistable perception? Differences between binocular rivalry and ambiguous figures. Journal of Vision, 4, 539-551.

Moreno-Bote, R., Rinzel, J., \& Rubin, N. (2007). Noise-induced alternations in an attractor network model of perceptual bistability. Journal of Neurophysiology, 98, 1125-1139.

Moreno-Bote, R., Shpiro, A., Rinzel, J., \& Rubin, N. (2010). Alternation rate in perceptual bistablity is maximal at and symmetric around equi-dominance. Journal of Vision, 10(11), 1-18.

Mueller, T. J., \& Blake, R. (1989). A fresh look at the temporal dynamics of binocular rivalry. Biological Cybernetics, 61, 223-232.

Nawrot, M., \& Blake, R. (1989). Neural integration of information specifying structure from stereopsis and motion. Science, 244, 716-718.

Necker, L. A. (1832). Observations on some remarkable optical phaenomena seen in Switzerland; and on an optical phenomenon which occurs on viewing a figure of a crystal or geometrical solid. London and Edinburgh Philosophical Magazine and Journal of Science, 1, 329-337.

Pastukhov, A., \& Braun, J. (2011). Cumulative history quantifies the role of neural adaptation in mulitstable perception. Journal of Vision, 11(10), 1-10.

Petersik, J. T., Shepard, A., \& Malsch, R. (1984). A three-dimensional motion aftereffect produced by prolonged adaptation to a rotation stimulation. Perception, 13, 489-497.

Rock, I., \& Mitchener, K. (1992). Further evidence of failure of reversal of ambiguous figures by uninformed subjects. Perception, 21, 3945.

Stollenwerk, L., \& Bode, M. (2003). Lateral neural model of binocular rivalry. Neural Computation, 15, 2863-2882.

Struber, D., \& Stadler, M. (1999). Differences in top-down influences on the reversal rate of different categories of reversible figures. Perception, 28, 1185-1196.

Taylor, M. M., \& Aldridge, K. D. (1974). Stochastic processes in reversing figure perception. Perception \& Psychophysics, 16, 9-27.

Tong, F. (2001). Competing theories of binocular rivalry: A possible resolution. Brain and Mind, 2, 55-83.

Tong, F., \& Engel, S. E. (2001). Interocular rivalry revealed in the human cortical blind-spot representation. Nature, 411, 195-199.

Toppino, T. C. (2003). Reversible-figure perception: Mechanisms of intentional control. Perception \& Psychophysics, 65, $1285-1295$.

Toppino, T. C., \& Long, G. M. (1987). Selective adaptation with reversible figures: Don't change that channel. Perception \& Psychophysics, 42, 37-48.

Toppino, T. C., \& Long, G. M. (2005). Top-down and bottom-up processes in the perception of reversible figures: Toward a hybrid model. In N. Ohta, C. M. MacLeod, \& B. Uttl (Eds.), Dynamic cognitive processes (pp. 37-58). Tokyo: Springer. 
van Ee, R., van Dam, L. C. J., \& Brouwer, G. J. (2005). Voluntary control and the dynamics of perceptual bi-stability. Vision Research, 45, 41-55.

Virsu, V. (1975). Determination of perspective reversals. Nature, 257, 786-787.

Von Grunau, M. W., Wiggin, S., \& Reed, M. (1984). The local character of perspective organization. Perception \& Psychophysics, 35, 319324.
Walker, P. (1975). Stochastic properties of binocular rivalry alternations. Perception \& Psychophysics, 18, 467-473.

Wallis, G., \& Ringelhan, S. (2013). The dynamics of perceptual rivalry in bistable and tristable perception. Journal of Vision, 13(2):24, 1-21.

Wilson, H. R. (2003). Computational evidence for a rivalry hierarchy in vision. Proceedings of the National Academy of Sciences of the USA, 100, 14499-14503. 